\title{
Temporal Artery Biopsy Roulette: Playing to Win
}

\author{
Kyle Swerhun ${ }^{1}$, Thomas Cousineau ${ }^{2}$, Sanjoy K Gupta ${ }^{3 *}$ \\ ${ }^{1}$ Department of Biology, Lakehead University, Thunder Bay, ON, Canada \\ ${ }^{2} \mathrm{OD}$, Cousineau Eye Centre, Fort Frances, ON, Canada \\ ${ }^{3}$ Associate Professor (Clinical Sciences), Northern Ontario School of Medicine, Lakehead University, Thunder \\ Bay, ON, Canada \\ "Corresponding Author: Sanjoy K Gupta, MD, PhD, FRCSC, Associate Professor (Clinical Sciences), \\ Northern Ontario School of Medicine, Lakehead University, Thunder Bay, ON, Canada, \\ Email: sgupta@tbaytel.net
}

Received Date: 29-01-2021; Accepted Date: 17-02-2021; Published Date: 25-02-2021

Copyright $^{\circledR} 2021$ by Swerhun K, et al. All rights reserved. This is an open access article distributed under the terms of the Creative Commons Attribution License, which permits unrestricted use, distribution, and reproduction in any medium, provided the original author and source are credited.

\begin{abstract}
Giant Cell Arteritis (GCA) is an auto inflammatory response often found in the superficial temporal artery. Patients may present with a localized headache overlying the temporal artery, as well as other symptoms such as weight loss, jaw claudication, shoulder stiffness and vision loss. Visual impairment from giant cell arteritis, associated with ischemic damage to the optic nerve or central retinal artery, may be irreversible. The diagnosis of giant cell arteritis may be confirmed by the presence of multinucleated giant cells in a temporal artery biopsy.
\end{abstract}

We present two case reports of patients with suspected GCA where diagnosis and management were complicated by delayed/negative temporal biopsies. We suggest a practical algorithm for the management of patients with suspected GCA.

\section{Keywords}

Auto Inflammatory; Giant Cell Arteritis; Erythrocyte Sedimentation Rate 


\section{Introduction}

Giant Cell Arteritis (GCA) is an inflammatory condition that develops in the inner linings of arterial walls. GCA is a systemic granulomatous auto inflammatory response on the internal elastic lamina of medium and large sized arteries [1,2]. The superficial temporal artery is a common site of arteritis associated with GCA and is of major concern when considering the symptoms and diagnosis of the disease [2]. Multinucleated giant cells can be found within the affected arteries and are the result of T-cells fusing with activated macrophages [3]. This inflammatory reaction may cause arterial occlusion which can induce ischemic damage to the optic nerve or the retina if specifically located in the central retinal artery $[1,3]$.

GCA can present with a multitude of symptoms. Patients at risk for GCA are typically women of European descent above the age of 50 [2]. The most common symptoms include headache, tenderness of the scalp, claudication of the jaw, and weight loss [1]. Vision-related complications arise suddenly and for the most part are experienced upon waking up in the morning. The specific visual symptoms associated with GCA are onset of double vision, blurred vision, and or complete vision loss in one or both eyes [4].

The gold standard in the diagnosis of GCA, is a Temporal Artery Biopsy (TAB). The TAB confirms whether inflammation is occurring in the artery and if there is a site of occlusion [5]. Other ways of diagnosing GCA include bloodwork and imaging techniques. C-Reactive Protein (CRP) and Erythrocyte Sedimentation Rate (ESR) levels may be elevated, although these are non-specific. An ultrasound can be carried prior to a TAB in order to detect arterial stenosis or obstruction [6]. A highly sensitive and specific ultrasound method used in the detection of GCA related abnormalities in the temporal and aortic arteries is Colour Doppler Ultrasonography (CDUS) [6].

Although there are guidelines regarding the best approaches to diagnose GCA, confirming a diagnosis is difficult. Elevated CRP or ESR levels may be caused by non-specific causes such as infection or inflammation unrelated to GCA [1]. For TABs, false negatives are a major problem as up to $30 \%$ of GCA patients may have negative biopsies [7]. A TAB is deemed negative if there is no sign of inflammation of the temporal artery wall, no tears in the internal elastic lamina, or an absence of giant cells [5]. If the arterial biopsy is too small in length the inflamed portions can be missed due to the presence of skip lesions. Skip lesions are a point in an artery where no inflammation is observed [7]. To avoid error in GCA diagnosis associated with skip lesions, it is recommended that the excised portion of the temporal artery is at least at the optimal length of $20 \mathrm{~mm}$ long [7]. In the case of a negative TAB, a biopsy of the contralateral artery can be carried to diminish the probability of a false negative. Previous studies have shown that test sensitivity may be increased by $4-15 \%$ by a contralateral biopsy [2]. 


\section{Case Report}

Names of patients have been changed to protect privacy and maintain confidentiality.

\section{Case Report 1}

Robert, a male in his late 70's, was referred by an optometrist due to suspected bilateral disc edema. Robert had experienced rapid and painless vision loss in his right eye. His peripheral vision deteriorated first along with a prompt drop in central visual acuity. No weight loss, headaches, or jaw claudication was experienced by him. Upon first examination in the right and left eyes respectively, the visual acuity was limited to hand motion and 20/20. A Right Relative Afferent Pupillary Defect (RAPD) was also detected. The intraocular pressures recorded were 24 and $21 \mathrm{~mm} \mathrm{Hg}$ in the right and left eye respectively. A dilated examination was performed and revealed optic disc edema with hemorrhages (Fig. 1). Based on the previous findings, a presumptive diagnosis of ischemic optic neuropathy with or without arteritic etiology was made. Subsequent tests of Robert's ESR and Partial Thromboplastin Time (PTT) values were within normal ranges. Robert was treated with $1 \mathrm{~g}$ of solumedrol intravenously daily for 5 consecutive days. After the five day course of solumedrol, Robert's visual acuity in the right eye improved to 20/40. A TAB was carried out on the left temporal artery (size of biopsy, $13 \mathrm{~mm}$ ) and produced negative results. Due to visual improvement from the intravenous steroid course, Robert was prescribed oral steroid treatment. Robert had type II diabetes and was on an oral hypoglycemic agent. Unfortunately, Robert began to experience uncontrollable blood sugar levels along with congestive heart failure. Accordingly, the steroids were rapidly tapered off. The last visual test for Robert showed 20/100 vision in the right eye.

\section{Case Report 2}

Joan, a woman in her early 70's, presented at the emergency department in June of 2014 with signs of vision loss. Joan had been experiencing headaches for five days prior and woke up with vision loss in the right eye. The demonstrated visual acuity of Joan was limited to hand motion and 20/25 in the right and left eyes, respectively. She had obvious disc edema in the right eye and a normal appearing disc in the left eye (Fig. 1). She described right temporal headaches that were exasperated by head movements. She denied having night sweats, myalgias, or jaw claudication. Joan had a history of diabetes, smoking, depression, and sleep apnea. Joan was on medication for the diabetes (oral hypoglycemic agents and insulin) and anti-depressants. A CT scan was carried out in June of 2014 and was normal. Blood work indicated an ESR level of $51 \mathrm{~mm} / \mathrm{hr}$ on July $14^{\text {th }}, 2014$ which rose to $81 \mathrm{~mm} / \mathrm{hr}$ three days later. Further tests were done to rule out syphilis, tuberculosis, Lyme disease, Bartonella and B12 deficiency. All of these tests carried out came back negative. Infectious processes and malignancies were also addressed via a lumbar puncture. All findings related to the puncture were normal. 
Joan was placed on oral steroids (50 $\mathrm{mg}$ of prednisone/day) for one week but showed no improvement. This was decreased to $30 \mathrm{mg}$ prednisone/day for three weeks until it was eventually stopped completely due to exacerbation of her blood sugars. No TAB was performed during this period. When Joan was seen by one of the authors for a second opinion, the prospect of GCA was raised and a delayed TAB was carried out three months after original vision loss. The TAB was performed on the right temporal artery (size of biopsy, $12 \mathrm{~mm}$ ). A healed lesion and thickening of arterial media with an expansion via collagenous matrix with basophilic degeneration was discovered in the left temporal artery. These findings, along with no active inflammation or giant cells being found, indicated resolved temporal arteritis inflammation. Joan was then referred to a rheumatologist who suggested additional oral steroids and alternative immunosuppressant methotrexate. She declined both options for treatments.

Six months after her first episode of vision loss, she experienced vision loss in the left eye. The noted visual acuities at that point were being able to count fingers and 20/60 in the left and right eye accordingly. Upon inspection, a left eye RAPD and disc edema was discovered. Solumedrol was prescribed intravenously along with another treatment of prednisone. Joan came back in January of 2015 and had visual acuity readings of 20/60 and 20/80 in the left and right eye respectively. She was once again unwilling to continue steroid treatments at that time which resulted in the tapering off of the prednisone over the course of a month. She did not attend further appointments.

\section{Discussion}

Onset of GCA is characterized by indicators such as headaches, visual impairment, and tenderness of the scalp [1]. The most specific symptom used to diagnose GCA is jaw claudication [1]. Jaw claudication is felt during instances of mechanical movement [1]. These collective symptoms from a patient are enough to warrant tests to investigate the possibility of GCA. Amongst the tests, a TAB is considered the gold standard in the diagnosis of GCA [5].

TABs are an invasive procedure that must be warranted prior to being carried out. A TAB for this reason is only used to rule in or out the prospect of GCA. A diagnosis of GCA without a $\mathrm{TAB}$ result to provide definitive evidence has the potential to be harmful to the patient. Steroids are used to treat GCA but can cause adverse effects and or death through means of susceptibility to infection, and bleeding ulcers [8]. The severity of the steroidal implications varies based on age, with older patients experiencing more severe symptoms [8]. In relation to the case reports, both Robert and Joan fell too ill during the steroidal treatment due to adverse reactions associated with their diabetes. Along with the importance of ensuring GCA is backed by a positive result in a TAB, the timing of when to order the TAB is just as critical. The window of opportunity is within fourteen days. If the TAB is ordered too late, a concrete diagnosis may be lost. As seen with Joan, a healed granuloma was discovered in her left temporal artery. If the GCA had been diagnosed sooner with a positive TAB prior to steroids, other complications associated with the course of treatment for Joan could have been avoided. 
The quality and size of a TAB should also be adequate to rule out GCA. The excised portion of the temporal artery should be an optimal length of $20 \mathrm{~mm}$ and should be taken bilaterally from the patient to increase test sensitivity by $4-15 \%$ [2,7]. If a TAB is not carried out quick enough or at all by the medical professionals aiding a patient with potential symptoms of GCA, the chance for a definitive diagnosis is lost. Many alternatives exist for the gold standard TAB, including a promising form of ultrasound called CDUS [6]. CDUS is able to detect GCA in the temporal artery, and in large blood vessels such as the aorta and corresponding aortic arch branches [6]. CDUS sets itself apart from the TAB in many aspects. A TAB is highly invasive while CDUS allows medical professionals to test suspected cases of GCA non-invasively. With reference to GCA test results produced by a CDUS, there is a greater sensitivity (100\%), and similar specificity to that of TAB results.

In 1990, the American College of Rheumatology (ACR) published guidelines for diagnosis of GCA [9]. Three out of the five criteria are necessary for positive diagnosis. However, this can be achieved without a positive TAB [9]. A paper by Murchison suggests that a positive temporal artery biopsy should be a mandatory criterion in the diagnosis of GCA [9]. Keeping this in mind, we have stratified GCA suspects as low risk or high risk. Fig. 2 lists the remaining ACR criteria (excluding a TAB) that may be used to diagnose patients with GCA. We recommend that patients with one out of the 4 criteria (low risk of GCA) should undergo a unilateral tab on the affected side (Fig. 2). In contrast, patients with two out of the four criteria (high risk of GCA) should undergo a bilateral TAB (Fig. 2). It is important to rule out GCA with a negative $\mathrm{TAB}$, otherwise patients may be subjected to extensive corticosteroid treatment unnecessarily. It was found that $28 \%$ of patients in a study who had a negative TAB result still satisfied the ACR's requirements for a diagnosis of GCA [10]. Therefore, these biopsynegative patients would have been treated long-term with corticosteroids treatment [10]. 


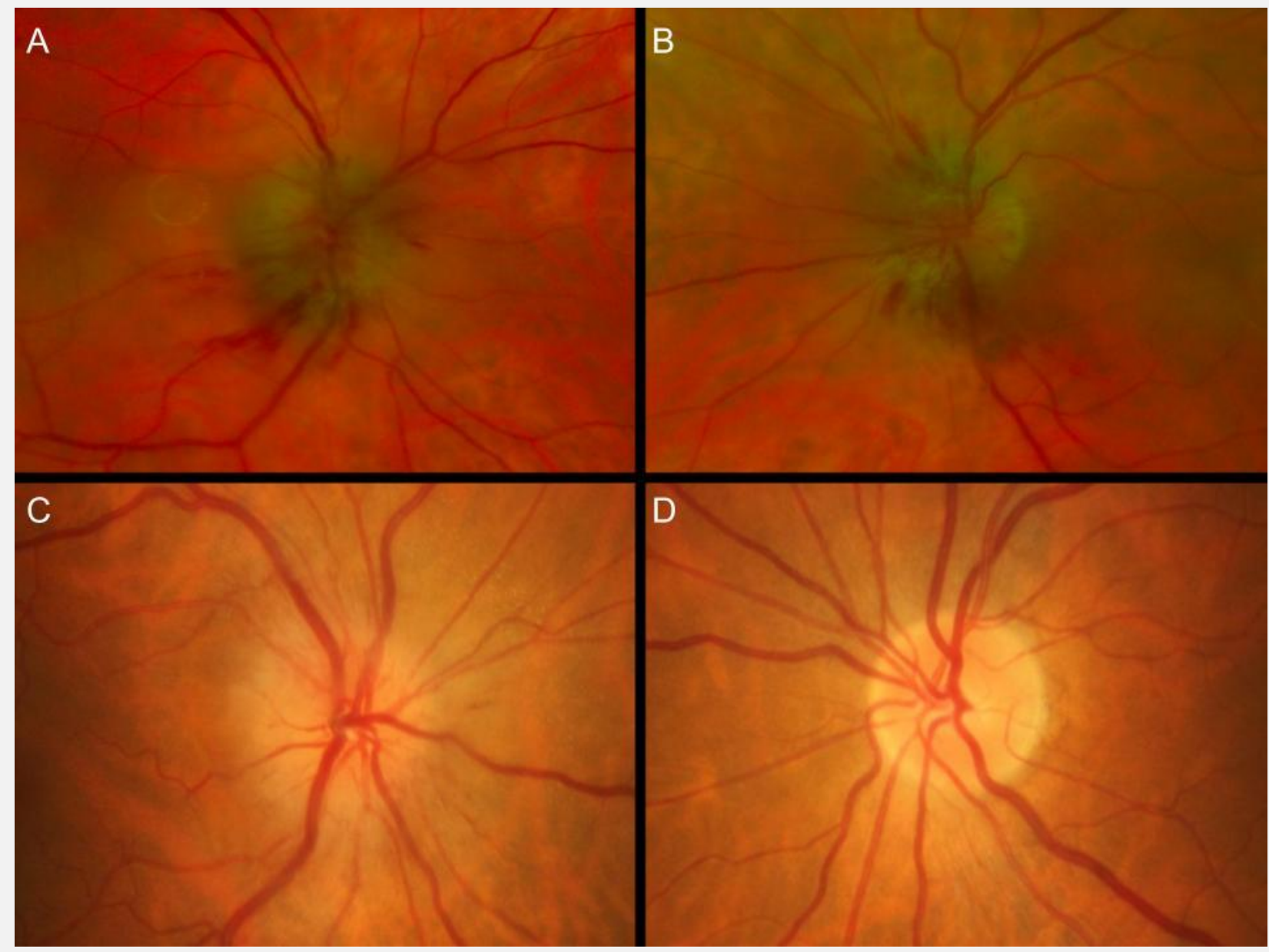

Figure 1: Optic disc images taken via a fundus camera from Robert and Joan are shown. Images A and B represent Robert's right and left eye accordingly. Images C and D represent Joan's right and left eye respectively. Image A shows an edematous optic disc with engorged blood vessels, an indication of inflammation of his right eye. The disc margin is blurred in all clock hours. Image B shows a disc hemorrhage and less severe disc edema. The temporal disc margin is still sharp. Image $C$ shows engorged blood vessels and optic disc edema with the disc margin blurred in all clock hours. Image D shows a normal optic disc and a sharp disc margin. 


\section{Risk Factors and Management Algorithm for suspected GCA patients}

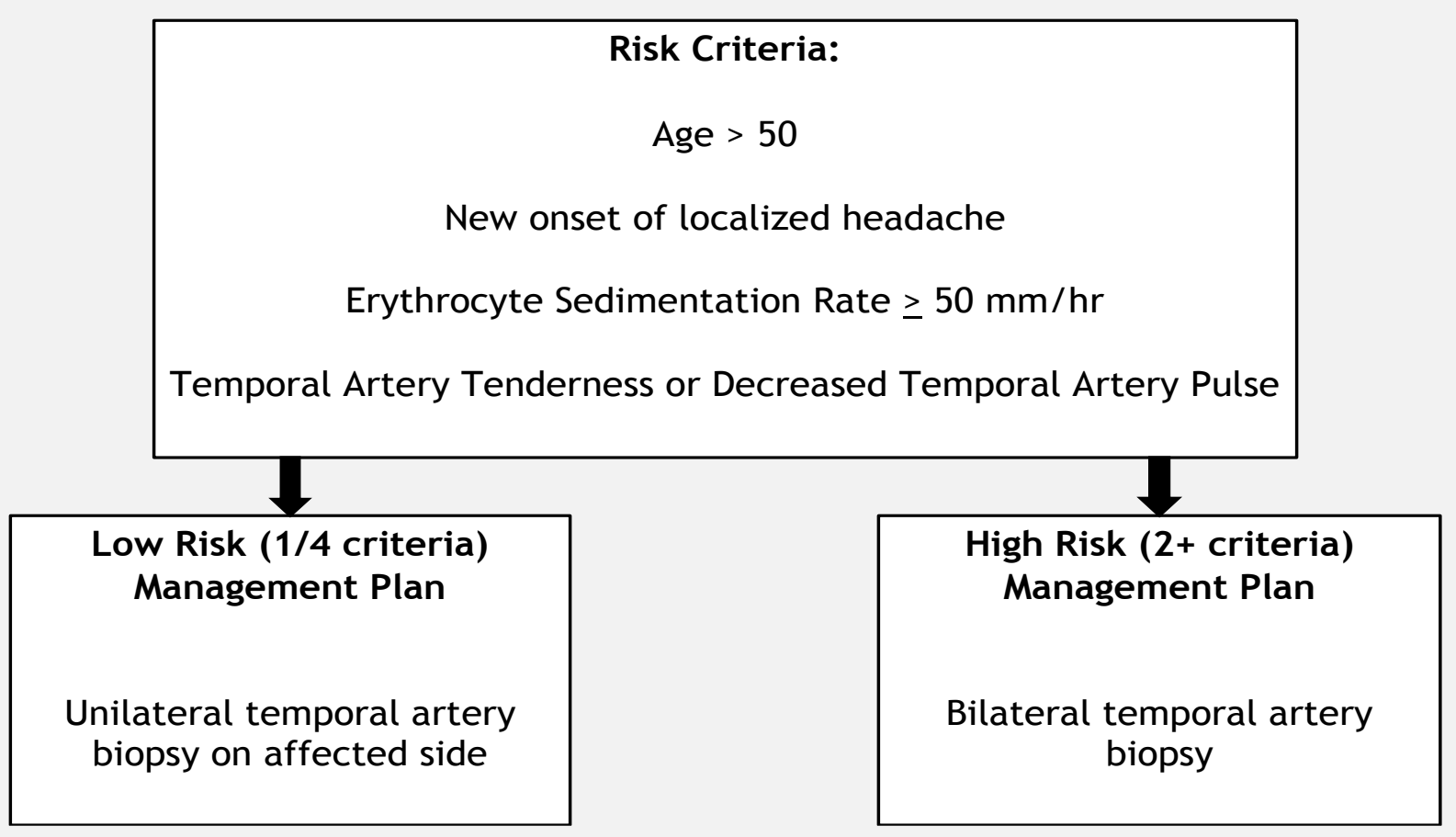

Figure 2: A practical management algorithm made with revisions to the American College of Rheumatology's GCA criteria. Patients with one of the risk factors are to be guided under the low risk GCA management plan. Patients with two or more of the risk factors are to be treated by following the high risk GCA management plan.

\section{Conclusion}

With all key ideas and concepts in mind, diagnosing a case of suspected GCA with highly sensitive and specific results is essential in providing optimal care for patients. Two testing methods that significantly fulfil both aspects include a TAB and a CDUS. Excising too small of a sample from a TAB, or not performing the test bilaterally increases the chance of a false negative test result. Due to the severity of the GCA, treatment needs to be carried out quickly and confidently following a thorough and bilateral TAB. The severity of GCA is especially apparent when considering the negative outcomes that are heightened by other existing health conditions, as seen in both reported cases. As a more sensitive and similarly specific alternative to the current gold standard TAB, a non-invasive CDUS may be carried out prior to a TAB in order to diagnose GCA with confidence. There may be a time in the future where it may be possible to diagnose GCA without an invasive procedure. 


\section{References}

1. Chacko JG, Chacko JA, Salter MW. Review of giant cell arteritis. Saudi J Ophthalmol. 2015;29(1):48-52.

2. Kale N, Eggenberger E. Diagnosis and management of giant cell arteritis: a review. Current Opinion in Ophthalmol. 2010;21(6):417-22.

3. Watanabe R, Goronzy JJ, Berry G, Liao YJ, Weyand CM. Giant cell arteritis: from pathogenesis to therapeutic management. Current Treatment Options in Rheumatol. 2016;2(2):126-37.

4. Vodopivec I, Rizzo III JF. Ophthalmic manifestations of giant cell arteritis. Rheumatol. 2018;57(2):63-72.

5. Kaltsonoudis E, Pelechas E, Papoudou-Bai A, Markatseli TE, Elisaf M, Voulgari PV, et al. The impact of temporal artery biopsy for the diagnosis of giant cell arteritis in clinical practice in a tertiary university hospital. Plos One. 2019;14(3):e0210845.

6. Diamantopoulos AP, Haugeberg G, Hetland H, Soldal DM, Bie R, Myklebust G. Diagnostic value of color Doppler ultrasonography of temporal arteries and large vessels in giant cell arteritis: a consecutive case series. Arthritis Care and Res. 2014;66(1):113-9.

7. Papadakis M, Kaptanis S, Kokkori-Steinbrecher A, Floros N, Schuster F, Hübner G. Temporal artery biopsy in the diagnosis of giant cell arteritis: Bigger is not always better. Am J Surg. 2018;215(4):647-50.

8. Nesher G, Sonnenblick M, Friedlander Y. Analysis of steroid related complications and mortality in temporal arteritis: a 15-year survey of 43 patients. J Rheumatol. 1994;21(7):1283-6.

9. Murchison AP, Gilbert ME, Bilyk JR, Eagle Jr RC, Pueyo V, Sergott RC, et al. Validity of the American college of rheumatology criteria for the diagnosis of giant cell arteritis. American J Ophthalmol. 2012;154(4):722-9.

10. Lee AW, Chen C, Cugati S. Temporal arteritis. Neurology: Clinical Practice. 2014;4(2):106-13. 